das Violett nach innen, das Roth nach aufsen. Wenn man statt des leuchtenden Punktes einen Spalt nimml, sieht man wieder das circulare Spectrum, wozu jetzt aber noch ein weifser Durchmesser kommt. Bei schiefer Stellung der Spalte und Linse gewabrt man noch andere, zum Theil recht schöne Bilder auf der Linse.

Achromatische Linsen zeigen wesentlich dasselbe, nur tritt die bekannte apfelgrüne Farbe statt der Spectralfarben auf.

Auf dieselbe Weise kann man auch die Diffractions. apparate beobachten; man stelle nur einen Spalt oder ein Fraunhofer'sches Gitter im convergirenden Lichte auf und betrachte es durch eine kleine Oeffnung in dem in der Bildfläche aufgestellten Schirme.

XV. Noch Einiges ïber Kohlrausch's Versuch zur Bestimmung des Verhätnisses der Wärmecapacitäten von Gasen; von L. Boltzmann.

Dieser Versuch verdankt Hrn. Witt e zwei Verbesserungen, die sich in der Folge als sehr nützlich erweisen dürften; nämlich das Manometer mit momentaner Communication und die Anwendung von Gefäfsen, die nicht wie Stiefel und Recipient der I uftpumpe durch einen verbältuifsmäfsig engen Kanal in Verbindung stehen. Was jedoch die Theorie des Versuches betrifft, so beging Hr. Wilte ein Versehen. Um das Verhăltuifs der Wärmecapacilăten zu bestimmen, denkt er sich nämlich (Pogg. Ann. Bd. 138, S. 158) ein Gas von der Temperatur $t^{\prime}$ bei constantem Drucke zur Temperatur $t$ erwärmt und dann ohne Wärmezufuhr auf sein ursprüngliches Volumen comprimirt. Die Temperatur soll dabei auf $t^{\prime \prime}$ steigen. Er glaubt, dafs man ihın hierbei im Ganzen dieselbe $W$ ărmemenge zuführen müsse, als wenn man es direct bei constanten Volum vou der Anfangs-Tem- 
peratur $t^{\prime}$ auf die Endtemperatur $t^{\prime \prime}$ erwärmen würde. Dieser Schlufs ist jedoch nicht erlaubt, da die zugeführte Wärne nicht blofs vom Anfangs - und Endszustande abhăngt. Die vollsländige Formel für das Verbältnifs der Wärmecapacitäten lautet vielmehr:

$$
\frac{c_{p}}{c_{c}}=\frac{\log p^{\prime}-\log p}{\log b-\log v^{\prime}}
$$

wobej $c_{p}$ die Wärmecapacität bei constanlem Drucke, $c_{c}$ die bei constantem Volumen, $p$ und $v$ Druck und Volum zu Anfang, $p^{\prime}$ und $v^{\prime}$ zu Ende des Processes sind. (Siehe Zeuner's Wärmelehre Seite 131). I)ie Formel Witte's gilt nur als Annäherungsformel für kleine Compressionen (denn bei kleiner Compression wird der Unterschied beider Wärmemengen klein von der zweiten Ordnung) und man kann aus ihr keineswegs den Schlufs ziehen, dafs $\frac{c_{n}}{c_{p}}$ Function des Druckes oder der Temperatur sey, wie es Witte in einer späteren Abhandlung (d. Ann. Bd. 140, S. 657 und 658) thut. Vielmehr machen sowohl theoretische Betrachtungen, als auch die Experimente Regnault's wahrscheinlich, dafs $\frac{c_{p}}{c}$ nahezu constant ist, wenn gleich eine absolute Constanz für die in der Natur vorkommenden Gase uatürlich nicht zu erwarten steht.

Die Ableitungsweise der Formel (1) setzt voraus, dafs der Kolben in jedem Momente unter demselben Drucke stehe, unter dem er stehen würde, wenn das Gas in dem Raume, den es augenblicklich erfüllt bei seiner (mittleren) Temperatur im Gleichgewicht wäre, dafs, wie man sich in der Wärmetheorie ausdrückt, der Druck gleich den Gegendruck ist. Ich habe nun nachgewiesen, dafs, wenn der vom Gase erfïllte Raum ein Cylinder ist, die Dichte der unmittelbar am Kolben anliegenden Gasschicht in jedem Augenblicke nur sehr wenig von der Dichte abweicht, die das Gas hătte, wenn es seinen Raum gleichförmig erfiillen würde; und da die Geschwindigkeit dieser Gasschicht gleich der Kolbengeschwindigkeit ist, so mufs auch der Druck, 
unter dem der Kolben steht, nahe gleich dem Druck seyn, den das Gas bei seinem augenblicklichen Volumen und Temperatur ausüben würde, folglich ist auch die Arbeit in beiden Fällen nahe dieselbe. Die bei Ableitung der Gleichung (1) gemachten Voraussetzungen sind also dann nahe erfüllt. Die Behauptung Kurz's, dafs hierbei eine Verwechslung von Druck und Arbeit stattfinde, beruht auf einem Mifsversländnisse. Wenn ich dagegen in meiner früheren Abhandlung sagte: -Es wäre ein grober Irrihum " usw., so behauptete ich damit nicht, dafs Hr. Kurz wirklich in diesen Irrthum verfallen sey. Ich meinte blofs, dafs man beim Lesen des Schlusses seiner Abhandlung leicht zu diesem Irrthume verleitet werden könne. Wenn Hr. Kurz blofs ausdrück en wollee, dafs der Verbindungscanal zwischen Stiefel und Recipient in Kohlrausch's Versuche so eng war, dafs unter dem Kolben nahezn ein Yacuum entsland und daher die im Recipienten befindliche Luft in den leeren Raum ausströmte, so kann ich dem um so weniger entgegentreten, als mir die $W_{\text {eite }}$ dieses Verbindungscanals durchaus unbekannt ist. Moglicherweise war diefs die $\mathbf{U r}$ -

sache, weshalb Kohlenrausch den Werth $\frac{c_{p}}{p_{r}}$ zu klein erhielt: nur weshalb die Theorie die Temperaturerhöhung tiberhaupt läugnen sollte, begriff ich nicht. Schliefslich erlaube ich mir, für den Versuch Kohlrausch's folgende Form vorzuschlagen:

Das Gas befinde sich in einem einzigen cylindrischen Gefäfs, in das ein luftdicht schliefsender Kolben bei einigen Versuchen tiefer, bei anderen weniger tief eingesenkt wird. Nahe am Bodep des Gefälses seyen mehre Manometer angehracht, um den Druck sowohl im Momente des Endes der Kolbenbewegunga als auch beliebige Zeit später bestimmen zu könnẹn. Kolben und Manoneterhähne werden durch eine Maschinerie getrieben, um nicht nur die gröfste Uebereinstimmung bei den einzelnen Versuchen erzielen, sondern auch die Art und Weise der Kolbenbewegung genar bestimmen zun können. Die Zeitmomenłe könnten tele- 
graphisch registrirt werden. Es ist dann der Druck in jedem Augenblicke nur wenig vom Gegendrucke verschieden und ihre Differenz könnte zu dem noch berechnet werden. Aufserdem könnte der Abkühlungscoëfficient für jedes Volumen des Gases besonders bestimmt werden. Auf diese Weise könnte vielleicht auch die Beantwortung der Frage versucht werden, wie rasch die Unselzung der progressiven Bewegung der Moleküle in Bewegung der Atome gegeneinander (sogenannte intramolekulare) geschieht. Es ist zwar nicht wahrscheinlich, aber immerhin nicht aufserhalb des Bereiches der Möglichleit, dafs diese Unsetzung eine längere Zeit beanspruche.

Graz, den 11. November 1870 .

\section{Eine pseudoskopische und optometrische} Figur; von H. Emsmann in Stettin.

A. Der nicht unbedeutenden Anzahl bekannter pseudoskopischer Figuren erlaube ich mir eine hinzuzufugen, welche sehr verschiedenartige Eindrücke hervorbringt und daher von besonderem Interesse erscheint.

Es besteht die Figur (Fig. 6 Taf. VI) aus drei gleichen neben einander liegenden Quadraten $(a)$, so dafs die Verlăngerungen der Seiten des mittelsten derselben Seiten der nebenliegenden bilden; an den Seiten der Quadrate liegen einerseits sechs, andererseits vier gleiche Parallelogramme $(b$ und $c)$ in der Richtung derjenigen Diagonalen der Quadrate, welche die aneinander stofsenden Quadrate nicht verbinden; die Parallelogramme können leer oder durch parallele, in gleichem Abstande gezogene Linien, wie die Figur zeigt, getheilt seyn.

Fixirt man die Mitte der Figur und zwar am besten mit nur einem Auge, weil dann die Täuschung leichter, als 